\title{
Monte Carlo methods for estimating depletion potentials in highly size- asymmetrical hard sphere mixtures
}

Cite as: J. Chem. Phys. 139, 144102 (2013); https://doi.org/10.1063/1.4824137

Submitted: 25 June 2013 . Accepted: 19 September 2013 . Published Online: 08 October 2013

D. J. Ashton, V. Sánchez-Gil, and N. B. Wilding
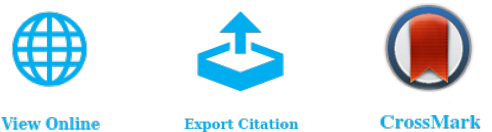

\section{ARTICLES YOU MAY BE INTERESTED IN}

Monte Carlo cluster algorithm for fluid phase transitions in highly size-asymmetrical binary mixtures

The Journal of Chemical Physics 133, 194102 (2010); https://doi.org/10.1063/1.3495996

Perspective: The Asakura Oosawa model: A colloid prototype for bulk and interfacial phase behavior

The Journal of Chemical Physics 141, 140901 (2014); https://doi.org/10.1063/1.4896943

Demixing transition, structure, and depletion forces in binary mixtures of hard-spheres: The role of bridge functions

The Journal of Chemical Physics 139, 104908 (2013); https://doi.org/10.1063/1.4820559

Challenge us.

What are your needs for periodic signal detection?

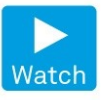

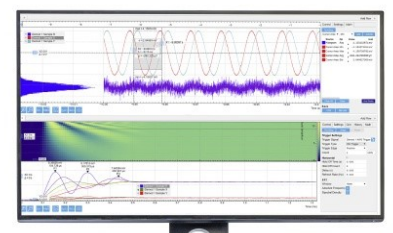

O
- Zurich

Instruments 


\title{
Monte Carlo methods for estimating depletion potentials in highly size-asymmetrical hard sphere mixtures
}

\author{
D. J. Ashton, ${ }^{1}$ V. Sánchez-Gil, ${ }^{2}$ and N. B. Wilding ${ }^{1}$ \\ ${ }^{1}$ Department of Physics, University of Bath, Bath BA2 7AY, United Kingdom \\ ${ }^{2}$ Instituto de Química Física Rocasolano, CSIC, Serrano 119, E-28006 Madrid, Spain
}

(Received 25 June 2013; accepted 19 September 2013; published online 8 October 2013)

\begin{abstract}
We investigate Monte Carlo simulation strategies for determining the effective ("depletion") potential between a pair of hard spheres immersed in a dense sea of much smaller hard spheres. Two routes to the depletion potential are considered. The first is based on estimates of the insertion probability of one big sphere in the presence of the other; we describe and compare three such methods. The second route exploits collective (cluster) updating to sample the depletion potential as a function of the separation of the big particles; we describe two such methods. For both routes, we find that the sampling efficiency at high densities of small particles can be enhanced considerably by exploiting "geometrical shortcuts" that focus the computational effort on a subset of small particles. All the methods we describe are readily extendable to particles interacting via arbitrary potentials. (C) 2013 AIP Publishing LLC. [http://dx.doi.org/10.1063/1.4824137]
\end{abstract}

\section{INTRODUCTION}

Effective potentials arise in theories of complex multicomponent fluids such as colloidal suspensions or polymer solutions which comprise mixtures of big and small particles. For such a system, one seeks to integrate out from the full Hamiltonian the degrees of freedom of the small particles in order to obtain an effective Hamiltonian for the big particles. The motivation for doing so is to create an approximate, yet analytically tractable description of the true system in terms of a single component model of big particles. Unfortunately, obtaining the full effective Hamiltonian is a tall order. ${ }^{1,2}$ A first step in any theoretical treatment is therefore to determine the two-body effective potential between a single pair of the big particles in a sea of the smaller species. However, even this task is challenging when there exists a very large disparity in size between the particles as is common in suspensions containing a mixture of two sterically stabilized colloid species. Such systems are often modelled as a highly size-asymmetric binary mixture of hard spheres, for which the effective interactions arise from the celebrated depletion mechanism. ${ }^{3}$ As well as being a key ingredient in determining effective Hamiltonians for asymmetrical hard sphere mixtures, depletion interactions can be directly measured in experiments. $^{4}$

For a pair of big hard spheres in a sea of small hard spheres, the effective potential takes the form

$$
\phi_{\mathrm{eff}}\left(r_{\mathrm{bb}}\right)=\phi_{\mathrm{bb}}\left(r_{\mathrm{bb}}\right)+W\left(r_{\mathrm{bb}}\right)
$$

where $\phi_{\mathrm{bb}}\left(r_{\mathrm{bb}}\right)$ is the bare hard sphere potential between two big spheres of diameter $\sigma_{b}$ whose centers are separated by a distance $r_{\mathrm{bb}}$, and $W$ is the "depletion potential" which is mediated by the small spheres of diameter $\sigma_{\mathrm{s}}$. In this paper we consider additive hard sphere mixtures so that the big-small interaction diameter $\sigma_{\mathrm{bs}}=\left(\sigma_{\mathrm{b}}+\sigma_{\mathrm{s}}\right) / 2$. In that case the depletion potential is attractive for small separations of the big spheres, but decays in an exponentially damped oscillatory fashion at large separations. The physics of the attraction is well understood: The exclusion or depletion of the small spheres as the big ones come close together results in an increase in free volume available to the small species leading to a net increase of entropy. ${ }^{3}$

A number of theoretical prescriptions exist for determining effective potentials, including integral equations (as summarized in the recent article by Boțan et al. ${ }^{5}$ ), density functional theory (DFT) (see Refs. 6 and 7 and the summary in Ashton et $a l .{ }^{8}$ ), and morphometric theory. ${ }^{5,9}$ However, these theoretical treatments involve approximations, the validity of which need to be checked. Indeed, even ostensibly small differences in the predictions for depletion potentials arising from the various theories can leads to substantial differences in the predictions of phase behaviour, particularly at higher volume fractions of the small particles. This was highlighted in Ashton et $a l .^{8}$ who compared values of the second virial coefficient of the depletion potential arising from a number of different theoretical approaches. Knowledge of this quantity allows one to predict the small particle volume fraction at which the fluid-fluid critical point occurs in the effective onecomponent fluid. Fairly substantial differences were found between the predictions of the various theories for the location of the critical point, at least for moderate size ratios. We note also that for the full mixture, the existence of such a critical point is a matter of longstanding debate, see, e.g., Refs. 10 and 11 and references therein. However, if it occurs at all, the critical point seems certain to be located at quite high volume fractions of the small particles and to be metastable with respect to freezing. ${ }^{12}$

Thus there is a need for methods that provide accurate information on highly size asymmetrical mixtures at high volume fractions of the small particles. Simulation potentially provides a route to estimating effective potentials which is in principle exact, and can therefore be used to verify theoretical predictions. Unfortunately, like analytical theory, it too finds the regime of large size asymmetry extremely challenging. 
The difficulty stems from the slow relaxation of the big particles caused by the presence of the small ones. Specifically, in order to relax, a big particle must diffuse a distance of order its own diameter $\sigma_{b}$. However, for small size ratios, $q$ $\equiv \sigma_{s} / \sigma_{b}$, and even at quite low volume fractions of small particles, many small particles will typically occupy the space surrounding a big particle and these hem it in, greatly hindering its movement. In computational terms this issue mandates a very small molecular dynamics timestep in order to control integration errors, while in basic Monte Carlo (MC), a very small trial step-size must be used in order to maintain a reasonable acceptance rate. Consequently, the computational cost of simulating highly size asymmetric mixtures by traditional means is prohibitive at all but very low volume fractions of small particles.

Owing to these difficulties, most previous simulation studies of highly size asymmetrical $(q \lesssim 0.1)$ hard sphere mixtures ${ }^{13-16}$ have adopted an indirect route to measuring depletion potentials based on measurements of interparticle force. The strategy rests on the observation that the force between two big particles can be expressed in terms of the contact density of small particles at the surface of the big ones. ${ }^{14,17}$ By integrating this (angularly dependent) contact density for fixed separation $r_{\mathrm{bb}}$ of the big particles and repeating for separations ranging from contact, $r_{\mathrm{bb}}=\sigma_{\mathrm{b}}$, to $r_{\mathrm{bb}}=\infty$, one obtains the force profile. This can in turn be integrated to yield an estimate of the depletion potential. However, the statistical quality of the data obtained via this route is typically quite low, particularly at small $q \leq 0.1$ and high densities of small particles. This presumably reflects the difficulties of measuring contact densities accurately (which entails the extrapolation of data accumulated away from contact) and the errors inherent in numerical integration.

Only a few studies have attempted to measure the depletion potential directly for $q \leq 0.1$ - see Malherbe and Amokrane $^{18}$ for a hard sphere study and Luijten and coworkers ${ }^{19,20}$ for more general potentials. These studies deployed a cluster algorithm (to be described in Sec. V A) to deal with the problem of slow relaxation outlined above. However, this algorithm is limited in the range of particle volume fractions for which it will operate efficiently and thus there is a need for alternative approaches that extend this range to higher values.

An additional drawback of previous studies is that they have treated the small particles canonically rather than grand canonically. Doing so complicates comparison with theoretical studies which are typically formulated in terms of an infinite reservoir of small particles. It is also at variance with the common experimental situation of a depletant that is in equilibrium with a bulk reservoir.

In what follows we consider how Monte Carlo simulation can be used to obtain direct and accurate estimates of the depletion potential between two big hard spheres separated by a distance $r_{\mathrm{bb}}$ immersed in a dense sea of small particles at size ratio $q=0.1$. Our focus is on techniques that are formally exact and which therefore allow confident comparison with theoretical predictions. We investigate a range of such methods, discussing their implementation and assessing their relative utility; comparisons with theoretical predictions have appeared elsewhere. ${ }^{8}$

\section{SYSTEM SETUP}

The simulation setup that we consider for the measurement of depletion potentials is depicted in cross section in Fig. 1. It comprises a cuboidal periodic simulation box with dimensions $L_{x}=3.5 \sigma_{\mathrm{b}}, L_{y}=L_{z}=2.0 \sigma_{\mathrm{b}}$. This box accommodates two big hard spheres and a large number of small ones. (Though as described below, in some instances it will prove beneficial to take one of the big particles to be a hard shell.) Owing to the spherical symmetry of the depletion potential we can, without loss of generality, fix the center of one of the big particles at the origin, while constraining the center of the other to occupy points along the $x$-axis at $x=r_{\mathrm{bb}} \geq \sigma_{\mathrm{b}}$. The only exception to this arrangement is the cluster algorithm to be discussed separately in Sec. V A.

We set the size of the small particles to be $\sigma_{\mathrm{s}}=0.1 \sigma_{\mathrm{b}}$, i.e., $q=0.1$. We also elect to treat them grand canonically so that their total number fluctuates. Conceptually this corresponds to a colloidal system connected to a reservoir of depletant particles whose properties are parameterized in terms of either the reservoir volume fraction $\eta_{\mathrm{s}}^{r}=\pi \rho_{\mathrm{s}} \sigma_{\mathrm{s}}^{3} / 6$, (with $\rho_{s}=N_{\mathrm{s}} / V$ the reservoir number density) or equivalently the conjugate chemical potential $\mu_{s}^{r}$. In practical terms, use of the grand canonical ensemble aids relaxation of small particle configurations because particle transfers (insertions and deletions) can be performed very efficiently. However to utilize this ensemble one needs to know accurately the chemical potential corresponding to a given $\eta_{\mathrm{s}}^{r}$. We obtain this from the equation of state of Kolafa $\mathrm{et} \mathrm{al.},{ }^{21}$ which we have checked provides a highly accurate representation of grand canonical ensemble simulation data. Transfers of small particles are effected using a standard grand canonical approach. ${ }^{22}$ For the most part we consider the case of a rather high reservoir volume fraction of small particles, $\eta_{\mathrm{s}}^{r}=0.32$, which also corresponds to the conditions depicted in the configurational snapshot of Fig. 1.

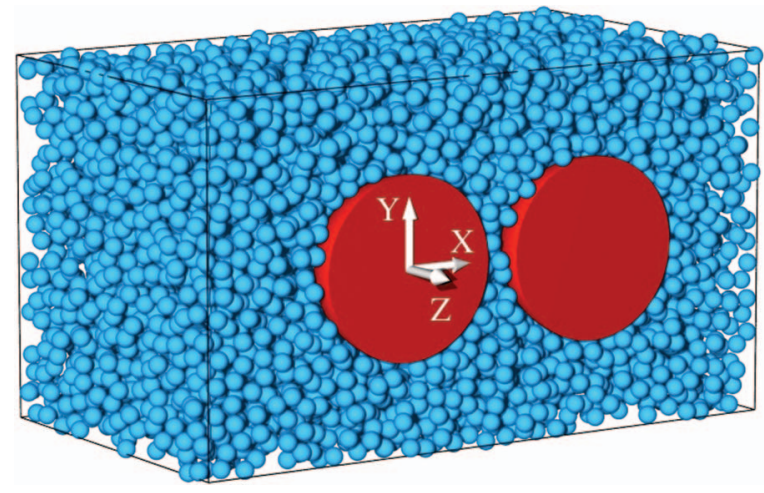

FIG. 1. A cross section through a snapshot of a configuration as described in the text. The simulation box contains a pair of big hard spheres, one of which is fixed at the origin, while the other is located at $x=r_{\mathrm{bb}}, y=0, z$ $=0$, with $r_{\mathrm{bb}}=1.19$ in this case. The big particles are in equilibrium with a fluid of small hard spheres (size ratio $q=0.1$ ) at reservoir volume fraction $\eta_{\mathrm{s}}^{r}=0.32$. The section shown corresponds to the region $z<0$. 


\section{OVERVIEW OF COMPUTATIONAL STRATEGIES}

We shall investigate two distinct routes to obtaining estimates of depletion potentials which we outline here before going into detail in Secs. IV and V. The first route is based on measurements of the insertion probability of one big sphere in the presence of the other; the second is based on direct sampling of free energy differences associated with variations in the separation between the two big spheres.

\section{A. Insertion route and the shell trick}

Let $\mu_{\mathrm{ex}}\left(r_{\mathrm{bb}}\right)$ be the excess chemical potential associated with inserting a big sphere at some prescribed distance $r_{\mathrm{bb}}$ from another big sphere. It is straightforward to show that this function is equivalent to the effective potential up to an additive constant, ${ }^{8,23}$ i.e.,

$$
W\left(r_{\mathrm{bb}}\right)=\mu_{\mathrm{ex}}\left(r_{\mathrm{bb}}\right)-C,
$$

where the constant

$$
C=\lim _{r_{\mathrm{bb}} \rightarrow \infty} \mu_{\mathrm{ex}}\left(r_{\mathrm{bb}}\right) .
$$

To facilitate estimates of the excess chemical potential, one can appeal to the Widom insertion formula, ${ }^{24}$ which in the case of hard particles reads

$$
\mu_{\mathrm{ex}}\left(r_{\mathrm{bb}}\right)=-\beta^{-1} \ln \left[p_{i}\left(r_{\mathrm{bb}}\right)\right] .
$$

Here, $p_{i}\left(r_{\mathrm{bb}}\right)$ is the probability that an attempt to insert a big particle at $x=r_{\mathrm{bb}}$ incurs no overlaps with small particles; it is calculated with respect to the ensemble of configurations of the small particles. $\beta$ is the inverse temperature, which in hard particle systems simply serves to bestow free energies with the appropriate dimensions; accordingly we shall henceforth set it to unity.

It follows from Eqs. (2)-(4) that the depletion potential can be expressed in terms of insertion probabilities as

$$
W\left(r_{\mathrm{bb}}\right)=\ln \left(\frac{p_{i}(\infty)}{p_{i}\left(r_{\mathrm{bb}}\right)}\right),
$$

where $p_{i}(\infty)$ represents the insertion probability for infinite separation of the big spheres, which in practical terms can be determined as the insertion probability of a big sphere in a simulation box containing only small particles.

The computational task is then to measure the insertion probability $p_{i}\left(r_{\mathrm{bb}}\right)$. Unfortunately, for the values of $\eta_{\mathrm{s}}^{r}$ of interest this probability is almost vanishingly small, a fact which renders simple sampling ineffective. Consequently we adopt a bespoke "gradual insertion" approach, based on the use of tunable interactions and biased Monte Carlo sampling. Details of this approach are postponed until Sec. IV. Here it suffices to note that in implementing such schemes for the special case of hard spheres, a very useful "geometrical shortcut" derives from the fact that it is not actually necessary to consider the insertion probability of a big hard sphere in order to calculate the depletion potential. Instead it is sufficient and (generally much more efficient) to measure the insertion probability for a hard shell of diameter $\sigma_{b}$ having infinitesimal thickness, as shown in the snapshot of Fig. 2. The essential observation is that when fully inserted, a hard shell particle en-

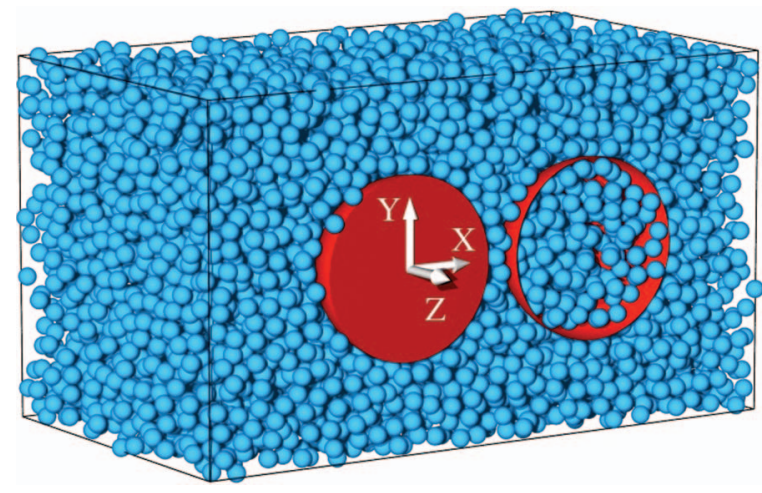

FIG. 2. A cross section through a configuration containing a big hard sphere fixed at the origin (left) and a fully inserted big hard shell (right) in equilibrium with a fluid of small particles at $\eta_{\mathrm{s}}^{r}=0.32$. The particle size ratio is $q=0.1$ and the separation is $r_{\mathrm{bb}}=1.19$. The section shown corresponds to $z<0$.

closes a number of small particles and although these remain in equilibrium with the reservoir (by means of particle transfers) they are fully screened from the rest of the system because their surfaces cannot penetrate the shell wall. Thus the contribution to the partition function from the enclosed particles is independent of $r_{\mathrm{bb}}$, and therefore represents a constant contribution to $\mu^{e x}\left(r_{\mathrm{bb}}\right)$ which vanishes from the difference in Eq. (2). Accordingly, Eq. (5) applies equally to shell insertion as it does to sphere insertion. Of course from a computational standpoint, the task of inserting a hard shell is much less challenging than that of inserting a hard sphere (as can be appreciated by comparing Figs. 1 and 2): essentially the insertion probability falls with the particle size ratio like $q^{2}$ rather than $q^{3}$. Shell insertion is deployed in each of the three gradual insertion methods to be described in Sec. IV.

A further geometrical shortcut results from noting that the convergence of the ensemble average over small particle configurations required to calculate the shell insertion probability depends on how quickly the small particles in the region of the shell decorrelate. To enhance this relaxation rate we preferentially perform grand canonical insertions and deletions of small particles within a shell subvolume of radius $0.7 \sigma_{b} \leq r \leq 1.3 \sigma_{b}$ centered on the shell. Updates inside the subvolume occur with a frequency 50-fold that of outside. This approach - which satisfies detailed balance - greatly reduces the time spent updating small particles whose coordinates are relatively unimportant for the quantity we wish to estimate.

\section{B. Direct sampling route}

The particle insertion approach outlined above relies on extracting the depletion potential from differences in the measured values of the insertion probability as a function of $r_{\mathrm{bb}}$. However, even when using the shell insertion trick, the difference $\ln p_{i}(\infty)-\ln p_{i}\left(r_{\mathrm{bb}}\right)$ that provides the depletion potential via Eq. (5), is (notwithstanding the logarithm) typically small compared to the absolute values of $\ln p_{i}(\infty)$ and $\ln p_{i}\left(r_{\mathrm{bb}}\right)$. Potentially, therefore, a great deal of computational effort is required to obtain a reasonable accuracy in $W\left(r_{\mathrm{bb}}\right)$. In view of 
this we have investigated an alternative strategy for obtaining the depletion potential which directly measures changes in the free energy as the separation between the two big spheres is varied. To achieve this, however, specialist methods are required to overcome the steric hindrance to the displacement of a big particle in a sea of much smaller ones. In Sec. V we consider two methods that enable such displacements via collective updates of a big sphere and many small ones. They are (i) the cluster algorithm of Dress and Krauth, ${ }^{25}$ which allows the depletion potential to be built up directly from the sampled histogram of big particle separations and (ii) a new constrained biased cluster move, which permits estimates of the free energy difference associated with a prescribed displacement of a big particle.

\section{INSERTION ROUTE: IMPLEMENTATIONS}

In this section we outline three methods that exploit the insertion route to determine the depletion potential. The basic idea is to fix a hard sphere at the origin and then estimate the probability of inserting a hard shell at coordinates $x=r_{\mathrm{bb}}, y=z=0$. In practice, however, for highly size asymmetrical mixtures and at all but the smallest values of $\eta_{\mathrm{s}}^{r}$, simple sampling of the insertion probability is too inefficient to yield accurate results. Instead a more elaborate gradual insertion technique is required to render the approach feasible. We note that key elements of the relevant strategies and general sampling issues for determining insertion probabilities (and thence excess chemical potentials) have been discussed previously elsewhere, ${ }^{26-30}$ though not in the context of highly size asymmetrical fluid mixtures.

\section{A. Method I: Expanded ensemble}

This method, which has been briefly reported previously, ${ }^{8}$ draws on earlier related studies. ${ }^{26-28,31}$ It involves defining an extended set of states for the interaction between the shell particle and the small particles and implementing Monte Carlo updates that make transitions between these states.

\section{Description}

To estimate $p_{i}\left(r_{\mathrm{bb}}\right)$ for the shell we suppose that it can exist in one of $M$ possible "ghost" states or "stages" in which it interacts with a small hard sphere (a distance $r_{\mathrm{bs}}$ away) via the potential

$$
\phi_{\mathrm{g}}^{(\mathrm{m})}\left(r_{\mathrm{bs}}\right)= \begin{cases}-\ln \lambda^{(\mathrm{m})}, & \left(\sigma_{\mathrm{b}}-\sigma_{\mathrm{s}}\right) / 2<r_{\mathrm{bs}}<\sigma_{\mathrm{bs}} \\ 0, & \text { otherwise }\end{cases}
$$

Here, $\mathrm{m}=0 \ldots M-1$ (an integer) indexes the stages, while the associated coupling parameter $0 \leq \lambda^{(\mathrm{m})} \leq 1$ controls the strength of the repulsion between the big particle and the small ones. Note that for $\lambda^{(\mathrm{m})}>0$ the repulsion is finite so that overlaps between small particles and the big one can occur. If we denote by $N_{\mathrm{o}}$ the instantaneous number of such overlaps, then the configurational energy associated with the shell in stage $m$ is

$$
\Phi_{\mathrm{g}}^{(\mathrm{m})}=-N_{\mathrm{o}} \ln \lambda^{(\mathrm{m})} .
$$

Clearly for $\lambda^{(\mathrm{m})}=1$, the shell is completely non-interacting, while for $\lambda^{(\mathrm{m})}=0$ it is infinitely repulsive. To span this range we set the extremal stages $\lambda^{(0)}=1$ and $\lambda^{(M-1)}=0$ (in fact we choose $\lambda^{(M-1)}=10^{-9}$ to avoid numerical infinities), and define a set of $M-2$ intermediate stages $\lambda^{(\mathrm{m})}, \mathrm{m}=1, \ldots$, $M-2$ that facilitate efficient MC sampling over the entire range $\mathrm{m}=0, \ldots, M-1$, i.e., that permits the shell interaction to fluctuate smoothly between the two extremes of interaction strength.

Details of a suitable Metropolis scheme for sampling the full range of $m=0 \ldots M-1$ have been described previously. ${ }^{28,32}$ The basic idea is to perform grand canonical simulation of the small particles, supplemented by MC updates that allow transitions $\mathrm{m} \rightarrow \mathrm{m}^{\prime}=\mathrm{m} \pm 1$ in the stage index. These transitions are accepted or rejected probabilistically on the basis of the change in the configurational energy, Eq. (7). Specifically

$$
p_{a}\left(m \rightarrow \mathrm{m}^{\prime}\right)=\min \left(1, \exp \left[-\left(\Phi_{\mathrm{g}}^{\left(\mathrm{m}^{\prime}\right)}-\Phi_{\mathrm{g}}^{(\mathrm{m})}\right)+\Delta w\right]\right),
$$

where $\Delta w=w^{\left(\mathrm{m}^{\prime}\right)}-w^{(\mathrm{m})}$, with $w^{(\mathrm{m})}$ a prescribed weight associated with stage $\mathrm{m}$ (see below). Note that for transitions that depart from the extremal stages $\mathrm{m}=0$ or $\mathrm{m}=M-1$, it is necessary to reject proposals that would take $m$ outside the range $(0, M-1)$.

The weights are chosen, as described below, such as to allow the system to smoothly sample the entire range of $\mathrm{m}$. Over the course of a sufficiently long run, the sampling results in the system visiting all the $M$ stages repeatedly, permitting a histogram $\tilde{H}(\mathrm{~m})$ of their relative probabilities to be accumulated. From this biased histogram, one unfolds the weight factors to obtain an estimate of the unbiased histogram:

$$
H(\mathrm{~m})=\tilde{H}(\mathrm{~m}) \exp \left(w^{(\mathrm{m})}\right) .
$$

After normalizing to unit integrated weight, this histogram provides an estimate of the relative probability $p\left(\mathrm{~m} \mid r_{\mathrm{bb}}\right)$ of finding the system in each of the $M$ stages. The insertion probability is simply the relative probability of finding the system in the extremal stages,

$$
p_{i}\left(r_{\mathrm{bb}}\right)=\frac{p\left(M-1 \mid r_{\mathrm{bb}}\right)}{p\left(0 \mid r_{\mathrm{bb}}\right)},
$$

from which the effective potential (up to a constant) follows via Eq. (5). Repeating the measurement for a succession of values of $r_{\mathrm{bb}}$ allows construction of the entire depletion potential.

\section{Remarks and results}

The implementation of method I entails a certain degree of preliminary work. First, one must decide on the number of stages $M$ and their locations in $\lambda \in[0,1)$, i.e., the set $\left\{\lambda^{(\mathrm{m})}\right\}$, $\mathrm{m}=1 \ldots M-2$ of intermediate stages that interpolate between the extremal values of $\lambda^{(0)}=1$ and $\lambda^{(M-1)}=10^{-9}$. It is important that these choices result in MC transitions $\mathrm{m}$ $\rightarrow \mathrm{m} \pm 1$ that are approximately equally likely in both directions and have a reasonably high rate of acceptance. To 
achieve this we perform a preliminary run in which we consider a single big ghost shell in the reservoir of small particles. We initially employ a large set of 1000 ghost stages, evenly spaced in $\ln \lambda$, and (in short runs) measure the distribution of overlaps $p\left(N_{\mathrm{o}} \mid \lambda^{(\mathrm{m})}\right)$ for each. From this set we select a subset of $M$ stages for which the acceptance rate for transitions $\mathrm{m} \rightarrow \mathrm{m} \pm 1$ is approximately $20 \%$. A convenient basis for making this selection is the following expression for the acceptance rate between states $\mathrm{m}$ and $m^{\prime}=\mathrm{m} \pm 1$ in an optimally biased system:

$$
\bar{p}_{a}\left(\mathrm{~m} \rightarrow \mathrm{m}^{\prime}\right)=\sum_{N_{\mathrm{o}}} P\left(N_{\mathrm{o}} \mid \lambda^{(\mathrm{m})}, r_{\mathrm{bb}}\right) \mathcal{M}\left(\mathrm{m} \rightarrow \mathrm{m}^{\prime}\right),
$$

with $\mathcal{M}\left(\mathrm{m} \rightarrow \mathrm{m}^{\prime}\right)$ the Metropolis function

$$
\begin{aligned}
\mathcal{M}(\mathrm{m} & \left.\rightarrow \mathrm{m}^{\prime}\right) \\
& =\min \left(1, \exp \left[-\left(\Phi_{\mathrm{g}}^{\left(\mathrm{m}^{\prime}\right)}-\Phi_{\mathrm{g}}^{(\mathrm{m})}\right)+F^{\left(\mathrm{m}^{\prime}\right)}-F^{(\mathrm{m})}\right]\right) .
\end{aligned}
$$

Here the free energy difference $F^{\left(\mathrm{m}^{\prime}\right)}-F^{(\mathrm{m})}$ serves to define the optimal weight (cf. Eq. (8)), which can be estimated using the well known Zwanzig formula: ${ }^{33}$

$$
\begin{aligned}
F^{\left(\mathrm{m}^{\prime}\right)}-F^{(\mathrm{m})} & =-\ln \left\langle\exp \left[-\left(\Phi_{g}^{\left(\mathrm{m}^{\prime}\right)}-\Phi_{g}^{(\mathrm{m})}\right)\right]\right\rangle_{\mathrm{m}, r_{\mathrm{bb}}} \\
& =-\ln \left(\sum_{N_{\mathrm{o}}}\left(\frac{\lambda^{(\mathrm{m})}}{\lambda^{\left(\mathrm{m}^{\prime}\right)}}\right)^{N_{\mathrm{o}}} P\left(N_{\mathrm{o}} \mid \lambda^{(\mathrm{m})}, r_{\mathrm{bb}}\right)\right) .
\end{aligned}
$$

Choosing a low acceptance rate leads to a smaller required number of stages $M$, while a large acceptance rate necessitates a correspondingly larger $M$ as noted previously in other contexts. ${ }^{34}$ Although we find empirically that the overall efficiency of the method is not particularly sensitive to the choice of acceptance rate (provided it lies in the range $10 \%-50 \%$ ), the $20 \%$ figure that we quote seems to strike a reasonable balance between the length of the sampling path required to span the $M$ stages and the transition rate.

The second preliminary task is to prescribe a suitable set of $M$ weights $\left\{w^{(m)}\right\}$ for use in the acceptance probability
Eq. (8). The role of these weights is to bias the acceptance rates such as to enhance the sampling of states of low probability. Generally speaking a suitable set of weights is one which ensures approximately uniform sampling of the $M$ stages. ${ }^{31}$ The weights can be determined using a variety of methods (including directly from Eq. (12)), though in practice we favor the Transition Matrix Monte Carlo (TMMC) method detailed in the Appendix. Note that having determined a suitable set of weights for one value of the big particle separation $r_{\mathrm{bb}}$, this set will (typically) perform adequately at all values of $r_{\mathrm{bb}}$ to be studied, at least provided the variations in the depletion potential are not too large, as is certainly the case for the range $\eta_{\mathrm{s}}^{r} \leq 0.32$ considered here. Similarly one does not have to choose a new set of $\left\{\lambda^{(m)}\right\}$ for each choice of the big particle separation $r_{\mathrm{bb}}$, a single choice performs adequately for all separations.

Fig. 3 shows data accumulated for $r_{\mathrm{bb}}=1.06$, $q=0.1, \eta_{\mathrm{s}}^{r}=0.32$. For this state point, $M=16$ stages were required to realize a $20 \%$ acceptance rate for transitions in $\mathrm{m}$. A portion of the time series resulting from the sampling of $m$ is shown in Fig. 3(a), giving an impression of the timescale over which the sampling covers the entire range. The estimates of the probability distribution $p\left(\lambda^{(\mathrm{m})} \mid r_{\mathrm{bb}}\right)$ that results from unfolding the weights from the measured histogram $\tilde{H}(\mathrm{~m})$ (cf. Eqs. (9) and (10)) is shown in Fig. 3(b). From this, the insertion probability can be read off directly; it is found to be $O\left(10^{-200}\right)$, demonstrating the scale of the depths in probability that the method allows one to plumb. The rationale for the extreme improbability of successfully inserting a shell without the support of biased sampling is to be found in Fig. 2, specifically in the tightness of the small particle packing at this value of $\eta_{\mathrm{s}}^{r}$.

Finally, in this subsection we remark that since the full depletion potential is built up from separate and independent measurements of the insertion probability at various values of $r_{\mathrm{bb}}$, there is the opportunity to exploit parallelism by farming out each measurement on multi-core processors.
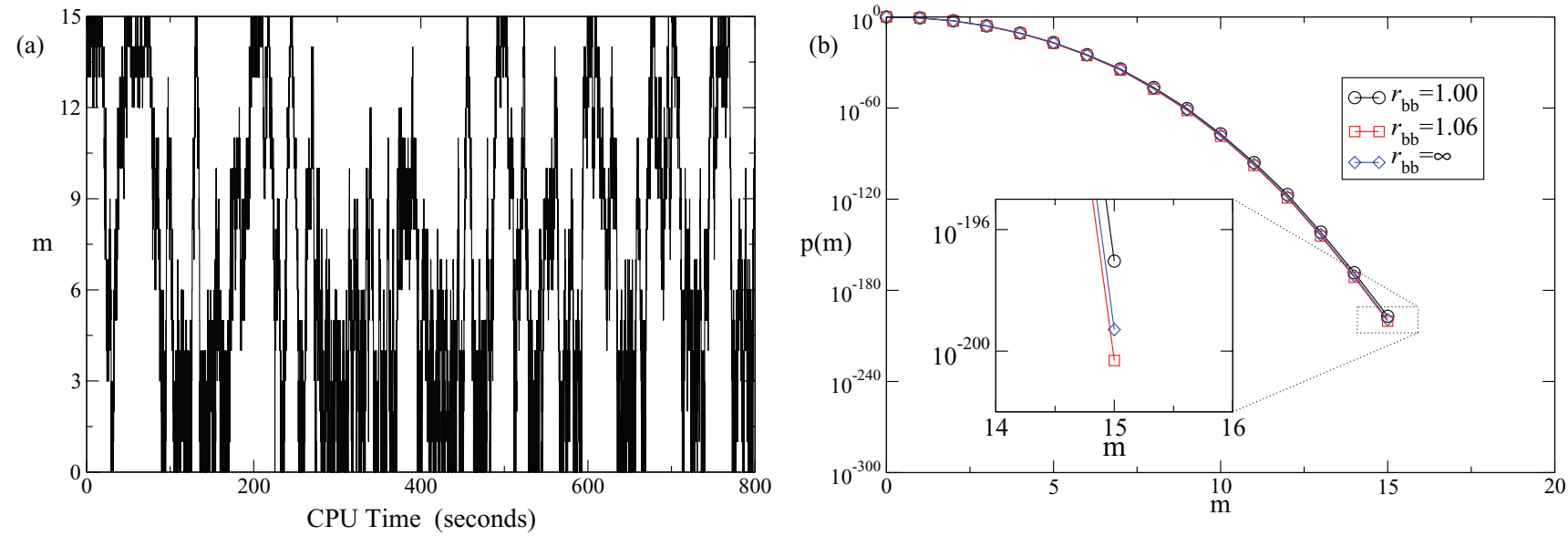

FIG. 3. (a) $\lambda^{(\mathrm{m})} v s \mathrm{CPU}$ time on a $2 \mathrm{GHz}$ processor for $r_{\mathrm{bb}}=1.06, \eta_{\mathrm{s}}^{r}=0.32, q=0.1$ as obtained for method I. The figure gives an impression of the typical time scale required to sample all $M=16$ stages, but constitutes only a small portion of the full run which comprised 35 CPU hours. (b) The unfolded histogram $p\left(\lambda^{(\mathrm{m})} \mid r_{\mathrm{bb}}\right)$ at a selection of values of $r_{\mathrm{bb}}$. Differences in the insertion probability $p\left(\lambda^{(M-1)} \mid r_{\mathrm{bb}}\right) / p\left(\lambda^{(0)} \mid r_{\mathrm{bb}}\right)$ (inset) provide estimates for the variations in the depletion potential. 


\section{B. Method II: Multiple overlapping histograms}

Our second approach is related to the previous one in that a set of $M$ stages are used to control the strength of interaction between the shell and the small particles in the manner described by Eq. (6). The difference is that here we do not actually implement transitions $\lambda^{(\mathrm{m})} \rightarrow \lambda^{(\mathrm{m} \pm 1)}$, instead we simply measure the free energy difference between successive values of $\lambda$.

\section{Description}

An accurate route to obtaining the difference in free energy between adjacent ghost stages is the well known formula of Bennett ${ }^{22,35,36}$

$$
F^{\left(\mathrm{m}^{\prime}\right)}-F^{(\mathrm{m})}=-\ln \left[\frac{\left\langle f\left(\Phi_{g}^{\left(\mathrm{m}^{\prime}\right)}-\Phi_{g}^{(\mathrm{m})}-C\right)\right\rangle_{\mathrm{m}, r_{\mathrm{bb}}}}{\left\langle f\left(\Phi_{g}^{(\mathrm{m})}-\Phi_{g}^{\left(\mathrm{m}^{\prime}\right)}+C\right)\right\rangle_{\mathrm{m}^{\prime}, r_{\mathrm{bb}}}}\right]+C .
$$

Here, $f(x)=1 /(1+\exp (x))$ and $C$ is an adjustable parameter. The ensemble averages in the numerator and denominator are performed with respect to the small particle configurations in stages $\mathrm{m}$ and $\mathrm{m}^{\prime}$, respectively, given a big particle separation $r_{\mathrm{bb}}$. These averages are obtained simply by summing over the relevant histogram, e.g.,

$$
\begin{aligned}
& \left\langle f\left(\Phi_{g}^{\left(\mathrm{m}^{\prime}\right)}-\Phi_{g}^{(\mathrm{m})}-C\right)\right\rangle_{\mathrm{m}, r_{\mathrm{bb}}} \\
& \quad=\sum_{N_{\mathrm{o}}} P\left(N_{\mathrm{o}} \mid \lambda^{(\mathrm{m})}, r_{\mathrm{bb}}\right) f\left(N_{\mathrm{o}}\left(\lambda^{(\mathrm{m})}-\lambda^{\left(\mathrm{m}^{\prime}\right)}\right)-C\right) .
\end{aligned}
$$

The optimal value of $C$ is the free energy difference itself. ${ }^{35}$ In practice one determines $C$ and $\Delta F=F^{\left(\mathrm{m}^{\prime}\right)}-F^{(\mathrm{m})}$ iteratively. Starting with $C=0$, Eq. (13) is used to estimate $\Delta F$, which serves as the new estimate for $C$ in a redetermination of $\Delta F$, and so on until $\Delta F$ converges.

Having chosen a suitable set of intermediate ghost stages $\left\{\lambda^{(\mathrm{m})}\right\}$, one simply measures the distribution of overlaps $P\left(N_{\mathrm{o}} \mid \lambda^{(\mathrm{m})}, r_{\mathrm{bb}}\right)$ at each $\lambda^{(\mathrm{m})}$. Bennett's method, Eq. (13), then yields the free energy differences between all the stages from which the insertion probability follows as

$$
\ln p_{i}\left(r_{\mathrm{bb}}\right)=F^{(0)}-F^{(M-1)} .
$$

The depletion potential is built up by repeating this measurement for a sequence of values of $r_{\mathrm{bb}}$ and utilizing Eq. (5) as was done in Sec. IV A.

\section{Remarks and results}

For this method to yield accurate results, stages should be placed at appropriate values of $\lambda$ such that successive distributions $p\left(N_{\mathrm{o}} \mid \lambda_{i}\right)$ and $p\left(N_{\mathrm{o}} \mid \lambda_{i+1}\right)$ overlap significantly. This is essentially the same criteria for choosing the set of intermediates $\left\{\lambda^{(\mathrm{m})}\right\}$ that is required to yield a reasonable acceptance rate between all stages in method I. Indeed the method described in Sec. IV A 2 for thinning out an initial choice of intermediate stages can be applied here too and the resulting set $\left\{\lambda^{(\mathrm{m})}\right\}$ is then equally applicable to methods I and II. We emphasize that for either method there is no need to recalculate the set $\left\{\lambda^{(\mathrm{m})}\right\}$ for each $r_{\mathrm{bb}}$ of interest; determining

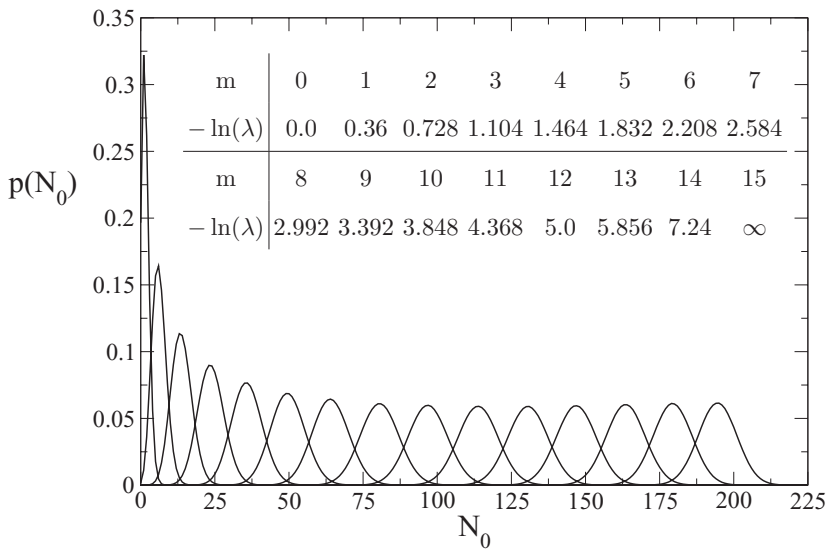

FIG. 4. The measured form of the overlapping distributions $p\left(N_{\mathrm{o}} \mid \lambda^{(\mathrm{m})}\right)$ employed to measure the shell insertion probability for $r_{\mathrm{bb}}=1.06$, $\eta_{\mathrm{s}}^{r}=0.32, q=0.1$ via method II. From right to left the distributions correspond to increasing $\mathrm{m}$ from $\mathrm{m}=0$ to $\mathrm{m}=14$. The corresponding values of $\ln \lambda$ are shown in the key. The distribution for $m=15$ is not depicted as it encompasses only the $N_{\mathrm{o}}=0$ state.

a set for one value of $r_{\mathrm{bb}}$ suffices for all values. We also remark in passing that while method II bears some resemblance to thermodynamic integration schemes, ${ }^{22}$ the estimates of the free energy differences are in principle exact-no numerical quadrature is involved.

Fig. 4 shows our measurements of the set of $M=16$ individual distributions $p\left(N_{\mathrm{o}} \mid \lambda^{(\mathrm{m})}\right)$ for $\eta_{\mathrm{s}}^{r}=0.32, q=0.1$ that yield an estimate of the insertion probability via application of Eqs. (13) and (15). The set $\left\{\lambda^{(\mathrm{m})}\right\}$ is the same as that used in method I and is listed in the key.

The chief merit of the multiple overlapping histogram approach compared to the expanded ensemble approach (method I) is its simplicity: no weights need to be calculated before one can start to accumulate data. Its main disadvantage compared to method $\mathrm{I}$, is the need to perform $M$ independent simulations and synthesize the results in a pairwise fashion. However, this drawback is somewhat mitigated by the fact that the independence of the simulations for each $\lambda^{(\mathrm{m})}$ renders them trivially parallel. Accordingly, one can farm out the calculations for each to a separate processor on a multiprocessor computer. Similarly the estimates of the insertion probability at the various values of $r_{\mathrm{bb}}$ that are needed to construct the full depletion potential are also independent, and can therefore be accumulated in parallel.

\section{Method III: Umbrella sampling}

This approach, which has some commonality with the umbrella sampling approach for calculating chemical potentials of Ding and Valleau, ${ }^{37}$ is conceptually simpler than the previous two in that it dispenses with staged intermediates.

\section{Description}

The algorithm considers an imaginary shell of diameter $\sigma_{b}$ centered on $x=r_{\mathrm{bb}}$. The instantaneous number of small particles, $N_{\mathrm{o}}$, that overlap this notional shell fluctuates with time, and hence one can measure its distribution $p\left(N_{\mathrm{o}} \mid r_{\mathrm{bb}}\right)$ as 
a histogram. Typically $N_{\mathrm{o}}$ will be large, but we can perform biased ("Umbrella") sampling with respect to insertion and deletion of the small particles in order to accurately measure the probability of states having $N_{\mathrm{o}}=0$. A little thought shows that this probability is just the shell insertion probability required for Eq. (5).

Operationally, transfers of small particles are performed according to the biased acceptance probabilities:

$$
\begin{aligned}
& p_{a}\left(N_{\mathrm{s}} \rightarrow N_{\mathrm{s}}+1\right)=\min \left(1, \frac{V}{N_{\mathrm{s}}+1} e^{\mu+W^{+}}\right), \\
& p_{a}\left(N_{\mathrm{s}} \rightarrow N_{\mathrm{s}}-1\right)=\min \left(1, \frac{N_{\mathrm{s}}}{V} e^{-\mu+W^{-}}\right) .
\end{aligned}
$$

These are the standard criteria for the grand canonical ensemble, ${ }^{22}$ modified by a weight factor $W^{ \pm}$that is non-zero if the proposed insertion or deletion of a small particle leads to a change in the number of overlaps $N_{\mathrm{o}}$. Specifically,

$$
\begin{aligned}
& W^{+}=w\left(N_{\mathrm{o}}\left(\{\mathbf{r}\}^{N_{s}+1}\right)\right)-w\left(N_{\mathrm{o}}\left(\{\mathbf{r}\}^{N_{\mathrm{s}}}\right)\right), \\
& W^{-}=w\left(N_{\mathrm{o}}\left(\{\mathbf{r}\}^{N_{s}-1}\right)\right)-w\left(N_{\mathrm{o}}\left(\{\mathbf{r}\}^{N_{\mathrm{s}}}\right)\right) .
\end{aligned}
$$

Here, $N_{\mathrm{o}}\left(\{\mathbf{r}\}^{N_{\mathrm{s}}}\right)$ is the number of overlap arising from the set of position vectors $\{\mathbf{r}\}^{N_{\mathrm{s}}}=\mathbf{r}_{1}, \mathbf{r}_{2} \ldots \mathbf{r}_{N_{s}}$ of $N_{\mathrm{s}}$ small particles, while $w\left(N_{\mathrm{o}}\right)$ is a weight function defined on the number of overlaps. These weights allow a single simulation run to sample not just the values of $N_{\mathrm{o}}$ that are typical for a given $\eta_{\mathrm{s}}^{r}$, but also the entire range down to $N_{\mathrm{o}}=0$. Accordingly one can measure a histogram of the weighted probabilities $\tilde{H}\left(N_{\mathrm{o}} \mid r_{\mathrm{bb}}\right)$, from which the Boltzmann histogram is obtained by unfolding the weights:

$$
H\left(N_{\mathrm{o}} \mid r_{\mathrm{bb}}\right)=\tilde{H}\left(N_{\mathrm{o}} \mid r_{\mathrm{bb}}\right) e^{w\left(N_{\mathrm{o}}\right)} .
$$

After normalization, this yields the probability distribution $p\left(N_{\mathrm{o}} \mid r_{\mathrm{bb}}\right)$, from which the insertion probability is read off as $p\left(N_{\mathrm{o}}=0 \mid r_{\mathrm{bb}}\right)$. The depletion potential (up to a constant) follows via Eq. (5). Repeating for a sequence of values of $r_{\mathrm{bb}}$ allows one to build up the entire depletion potential.

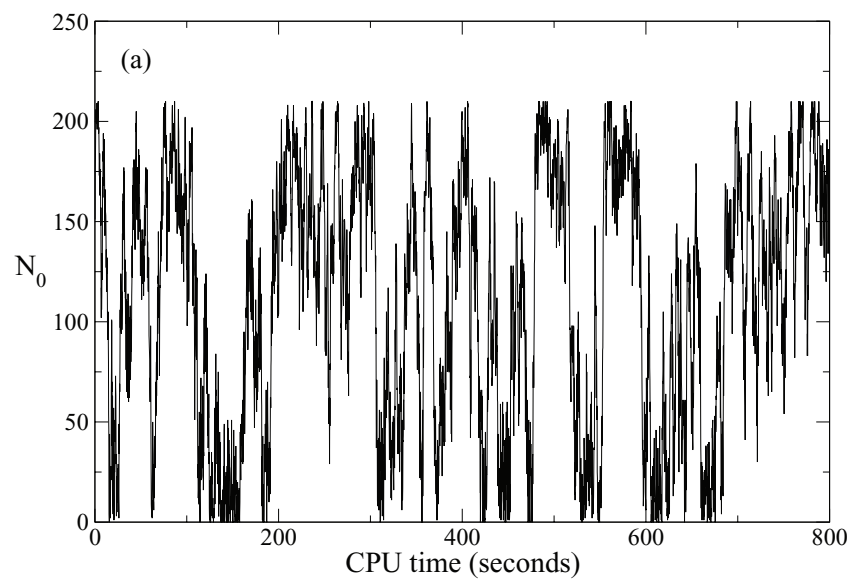

\section{Remarks and results}

As with method I, an appropriate set of weights is required for this method to operate effectively and again these can be readily determined using the TMMC method (see the Appendix). Fig. 5 shows a time series of the sampled values of $N_{\mathrm{o}}$ that results once the weights are in place. Owing to the biasing, the system samples smoothly the entire range from the most probable number of overlaps $N_{\mathrm{o}} \approx 200$, right down to $N_{\mathrm{o}}$ $=0$. The resulting form for $p\left(N_{\mathrm{o}} \mid r_{\mathrm{bb}}\right)$, obtained by unfolding the effects of the weights and normalizing the resulting histogram is shown in Fig. 5(b). From this one simply reads off the shell insertion probability as $p\left(N_{\mathrm{o}}=0 \mid r_{\mathrm{bb}}\right)$.

The chief merit of method III compared to methods I and II is that it is parameter free: there are no staged intermediates and therefore the associated inconvenience and startup costs of determining their number and appropriate placement are obviated. Nevertheless the computational cost of calculating weights represents a significant overhead as will be discussed in Sec. VI. We note that method III is parallelisable, but only with respect to the separate measurements at various $r_{\mathrm{bb}}$ needed to build up the depletion potential.

\section{DIRECT SAMPLING ROUTE: IMPLEMENTATIONS}

We now turn to consider two schemes that accumulate the depletion potential by focusing on the difference in effective potential as one varies $r_{\mathrm{bb}}$. They both rely on collective (cluster) updates of big and small particles. One is based on the cluster algorithm of Dress and Krauth, ${ }^{25}$ the other is a bespoke constrained cluster algorithm.

\section{A. Method IV: Geometrical cluster algorithm}

An efficient cluster algorithm capable of dealing with hard spheres mixtures was introduced by Dress and Krauth in $1995 .{ }^{25}$ It was subsequently generalized to arbitrary interaction potentials by Liu and Luijten ${ }^{19,38}$ who dubbed their method the Geometrical Cluster Algorithm (GCA).

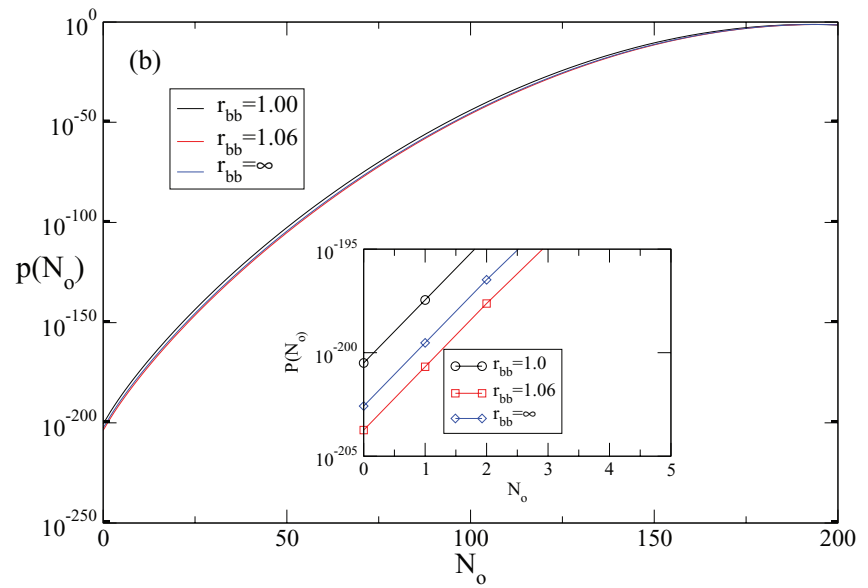

FIG. 5. (a) $N_{\mathrm{o}}(t) v s \mathrm{CPU}$ time on a $2 \mathrm{GHz}$ processor at $r_{\mathrm{bb}}=1.06 \sigma_{\mathrm{b}}, \eta_{s}=0.32, q=0.1$ as obtained from method III. The figure gives an impression of the typical timescale required to sample the range from $N_{\mathrm{o}} \approx 200$ to $N_{\mathrm{o}}=0$, but constitutes only a small portion of the full run which comprised $35 \mathrm{CPU}$ hours. (b) The form of the overlap probability distribution $p\left(N_{\mathrm{o}} \mid r_{\mathrm{bb}}\right)$ at three values of $r_{\mathrm{bb}}$. Differences in $p\left(N_{\mathrm{o}}=0 \mid r_{\mathrm{bb}}\right)$ as a function of $r_{\mathrm{bb}}$ (inset) yield the depletion potential as described in the text. 
A restricted Gibbs ensemble version of the GCA suitable for studying phase transitions was also subsequently developed. ${ }^{39-41}$ Here we describe the GCA for a general system of hard spheres in the canonical ensemble, before specializing to the case of a size asymmetrical binary mixture.

\section{Description}

The particles comprising the system are assumed to be contained in a periodically replicated cubic simulation box of volume $V$. The configuration space of these particles is explored via cluster updates, in which a subset of the particles known as the "cluster" is displaced via a point reflection operation in a randomly chosen pivot point. The cluster generally comprises both big and small particles and by virtue of the symmetry of the point reflection, members of the cluster retain their relative positions under the cluster move. Importantly, cluster moves are rejection-free even for arbitrary interparticle interactions. ${ }^{19}$ This is because the manner in which a cluster is built ensures that the new configuration is automatically Boltzmann distributed.

For hard spheres (there is no advantage in using shells in this context), the cluster is constructed as follows: one of the particles is chosen at random to be the seed particle of the cluster. This particle is point-reflected with respect to the pivot from its original position to a new position. However, in its new position, the seed particle may overlap with other particles. The identities of all such overlapping particles are recorded in a list or "stack." One then takes the topmost particle off the stack, and reflects its position with respect to the pivot. Any particles which overlap with this particle at its destination site are then added to the bottom of the stack. This process is repeated iteratively until the stack is empty and there are no more overlaps.

Note that cluster updates only displace particles, they do not allow their number to fluctuate. Accordingly, in order to treat the small particles grand canonically, we also perform insertions and deletions of small particles with a chemical potential corresponding to the prescribed $\eta_{\mathrm{s}}^{r}$, as outlined in Sec. II.

The effective potential $W(r)$ between two big particles is defined in terms of the radial distribution function $g\left(r_{\mathrm{bb}}\right)$, measured in the limit of infinite dilution:

$$
W(r)=-\lim _{\rho_{b} \rightarrow 0} \ln \left[g\left(r_{\mathrm{bb}}\right)\right],
$$

for $r_{\mathrm{bb}}>\sigma_{b}$. In our simulation studies this limit is approximated by placing a single pair of big hard spheres in the simulation box. A finite-size estimate to $g\left(r_{\mathrm{bb}}\right)$, which we shall denote $g_{L}\left(r_{\mathrm{bb}}\right)$, is then obtained by fixing the first of these particles at the origin and measuring (in the form of a histogram) the probability $p\left(r_{\mathrm{bb}}\right)$ of finding the second big particle in a shell of radius $r_{\mathrm{bb}} \rightarrow r_{\mathrm{bb}}+d r$. Then

$$
g_{L}\left(r_{\mathrm{bb}}\right)=\frac{p\left(r_{\mathrm{bb}}\right)}{p_{\mathrm{ig}}\left(r_{\mathrm{bb}}\right)},
$$

where the normalization relates to the probability of finding an ideal gas particle at this radius:

$$
p_{\mathrm{ig}}\left(r_{\mathrm{bb}}\right)=\frac{4 \pi r^{2}}{V} .
$$

To effect the measurement of $g_{L}\left(r_{\mathrm{bb}}\right)$, we modify the GCA slightly as follows: we choose one big particle to be the seed particle, which we place randomly within a shell $\sigma_{\mathrm{b}}<r_{\mathrm{bb}}<L / 2$, centered on the second big particle, with $L$ the linear box dimension. The location of the pivot is then inferred from the old and new positions of the seed particle. Thereafter clusters are built in the standard way. This strategy ensures that we efficiently sample separations of the big particles that lie in the range $\sigma_{\mathrm{b}}<r_{\mathrm{bb}}<L / 2$ for which $g\left(r_{\mathrm{bb}}\right)$ can sensibly be defined for hard spheres in a cubic box.

\section{Remarks and results}

For the systems of interest in this work, we find that the GCA is efficient for reservoir packing fractions $\eta_{\mathrm{s}}^{r} \leq 0.2$. Above this value, practically all the particles join the cluster, which merely results in a trivial point reflection of the entire system. Indeed the efficiency drop is so precipitous that $\eta_{\mathrm{s}}^{r}=0.2$ is the absolute upper bound on the volume fraction of small particles that can usefully be studied with this algorithm. For single component fluids this problem can be ameliorated by biasing the choice of pivot position to be close to the position of the seed particle. ${ }^{19}$ Doing so has been reported to extend the operating limit to $\eta_{\mathrm{s}}^{r} \simeq 0.34$. However, for the case of highly asymmetrical mixtures we find that this strategy does not significantly decrease the number of particles in the cluster because as soon as a big particle joins the cluster and is point reflected it causes many overlaps with small particles.

Fig. 6(a) shows the measured form of $g_{L}\left(r_{\mathrm{bb}}\right)$ for $\eta_{\mathrm{s}}^{r}=0.2, q=0.1$ obtained using a cubic simulation box of volume $V=\left(3 \sigma_{b}\right)^{3}$. For this measurement to provide an estimate of $W\left(r_{\mathrm{bb}}\right)$, it first has to be corrected for finite-size effects, manifest in the failure of the function to approach unity at large $r_{\mathrm{bb}}$. This is done (as has also been described elsewhere ${ }^{8}$ ) by measuring the cumulative integral

$$
G(R)=\int_{0}^{R} g_{L}(r) d r .
$$

This integral tends towards a smooth linear form quite rapidly as the upper limit $R$ increases. The measured limiting gradient, $\xi$, of $G(R)$ provides the requisite correction factor according to $g(r)=\xi^{-1} g_{L}\left(r_{\mathrm{bb}}\right)$. Following Eq. (19), the negative of the logarithm of $g(r)$ then yields an estimate for the effective potential $W\left(r_{\mathrm{bb}}\right)$, which is shown in Fig. 6(b).

The most attractive feature of the GCA for determining depletion potentials is that it allows direct sampling of the quantity of interest without the need for multiple simulations or biased sampling. Its principal drawback is that the method becomes unusable for $\eta_{\mathrm{s}}^{r} \gtrsim 0.2$, which limits its applicability. It is therefore of interest to consider whether one can formulate an algorithm that exploits the efficiency of collective updates, but operates at higher values of $\eta_{\mathrm{s}}^{r}$. The method 


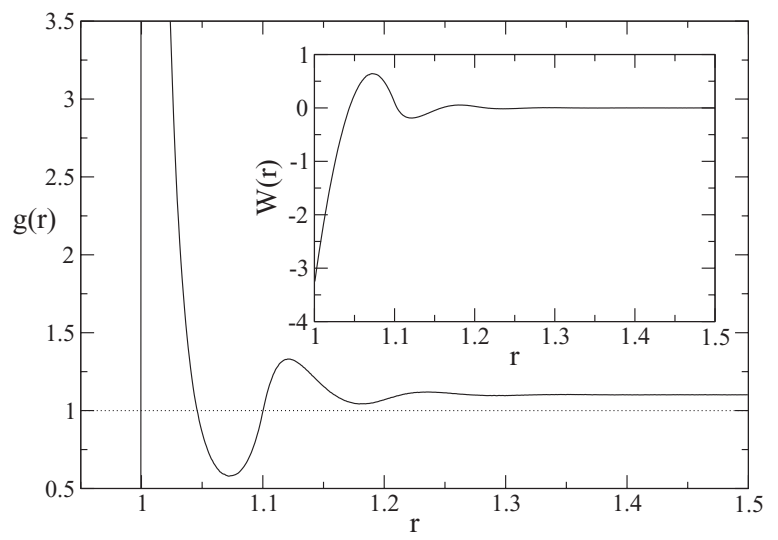

FIG. 6. The measured form of $g_{L}\left(r_{\mathrm{bb}}\right)$ corresponding to $\eta_{\mathrm{s}}^{r}=0.2, q=0.1$, obtained via method IV for a simulation box of dimensions $V=\left(3 \sigma_{b}\right)^{3}$. The limiting value differs from unity due to the finite-size effects described in Ashton et al. ${ }^{8}$ The inset shows the depletion potential $W(r)$ obtained by implementing the finite-size correction described in the text to $g_{L}\left(r_{\mathrm{bb}}\right)$ and applying Eq. (19).

described in Subsection V B achieves this, albeit at the expense of introducing biased sampling.

\section{B. Method V: Constrained cluster algorithm}

In common with the GCA, this method collectively moves a big hard sphere and a number of small ones via a self inverse operation. However, in contrast to the GCA it is a constrained scheme in the sense that it measures the free energy differences between two neighbouring discrete values of $r_{\mathrm{bb}}$.

\section{Description}

The operation of the method is shown schematically in Fig. 7. One big hard sphere (particle $A$ ) is fixed at the origin. The other (particle $B$ ) can occupy discrete values of $r_{\mathrm{bb}}$ set out along a one-dimensional radial grid which we take to be the $x$-axis. Let us label the grid points by the index $i$, and consider the situation when the big particle is stationed at $r_{\mathrm{bb}}=x_{i}$. We then estimate the free energy difference between grid points $i$ and $i+1$ in the following manner.

With particle $B$ stationed at grid point $i$, we equilibrate the small particles via transfers with the reservoir. For some equilibrium configuration of the small particles we then consider (but do not implement) a trial move to take particle $B$ from grid point $i$ to grid point $i+1$ as follows:

1. Reflect the center of particle $B$ in the plane normal to the $x$ axis which cuts the $x$ axis at $x=\left(x_{i}+x_{i+1}\right) / 2$. This takes particle $B$ from grid point $i$ to grid point $i+1$ as shown in Fig. 7(a).

2. Under this move, particle $B$ will overlap with a number $n$, say, of small particles. We then imagine reflecting these $n$ small particles in the same reflection plane. This switches them into the space left by particle $B$, see Fig. 7(a).

3. After undergoing this reflection, some of the $n$ small particles will overlap with other small particles or with the
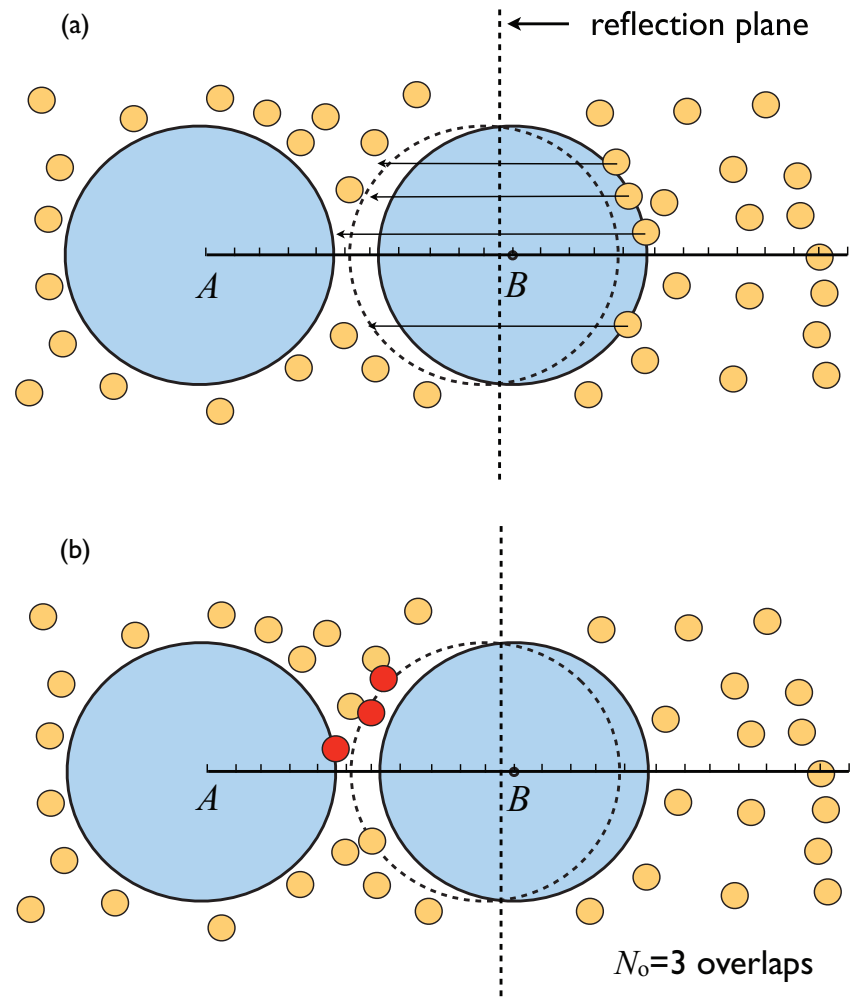

FIG. 7. Schematic illustration of the constrained cluster update of method V. (a) The big particle $B$ at $x_{i}$ undergoes a plane reflection to $x_{i+1}$ and thereby overlaps with $n$ small particles which are themselves subsequently reflected in the plane. (b) In their new position, the $n$ small particles overlap with $N_{\mathrm{o}}$ other particles (one of which may be the big particle $A$ at the origin). The number of such secondary overlaps $N_{\mathrm{o}}$ is the primary observable.

big particle $A$, as shown in Fig. 7(b). The number of such "secondary" overlaps is the observable $N_{\mathrm{o}}$ for the current configuration of small particles.

One then samples the fluctuations in $N_{\mathrm{o}}$ with respect to the ensemble of small particle configurations and accumulates its probability distribution $p\left(N_{\mathrm{o}}\right)$ as a histogram. Similarly to methods I-III, it is beneficial to preferentially implement transfers of small particles in a shell region around big particle $B$; this concentrates the computational effort on those regions which contribute most to the measurement. The sampling of the small particle configurations is biased so as to enhance the occurrence of values of $N_{\mathrm{o}}$ down to $N_{\mathrm{o}}=0$. This is achieved by defining a weight function $w\left(N_{\mathrm{o}}\right)$ which is incorporated in the GCE acceptance probabilities Eq. (16), in exactly the same manner as described for method III. An appropriate weight function can be found automatically using the TMMC method described in the Appendix.

Together these measures enable an efficient and accurate estimate for the probability that the trial collective move leads to $N_{\mathrm{o}}=0$, i.e., a valid hard sphere configuration. Let us denote this probability $p_{i}^{+}(0)$ because we have measured it with particle $B$ moving from grid point $i$ to $i+1$. Similarly we can measure the probability $p_{i+1}^{-}(0)$ that a move from $i+1 \rightarrow i$ leads to zero overlaps. Then the measured ratio $p_{i}^{+}(0) / p_{i+1}^{-}(0)$ provides the difference in the depletion potential between grid points $i$ and $i+1$ via an expression akin to Bennett's 
acceptance ratio formula: $:^{35}$

$$
W\left(x_{i+1}\right)-W\left(x_{i}\right)=\ln \frac{p_{i+1}^{-}(0)}{p_{i}^{+}(0)} .
$$

From measurements of the difference in the depletion potential between all neighbouring pairs of grid points, one extracts the depletion potential itself simply by summing, commencing at a value of $r_{\mathrm{bb}}$ sufficiently large that $W\left(r_{\mathrm{bb}}\right)$ can be considered to have decayed to zero.

\section{Remarks and results}

Compared to the GCA (method IV), the principal asset of method $\mathrm{V}$ is that it permits study of considerably larger volume fractions of the small particles. This is because the number of particles involved in the collective move is not allowed to grow indefinitely. Instead cluster growth is truncated after one iteration and biased sampling used to obtain the information required to estimate the depletion potential. We note that a constrained cluster algorithm suitable for estimating depletion potentials has previously been described by Malherbe and Krauth, ${ }^{42}$ however it does not truncate cluster growth and therefore is limited to much lower values of $\eta_{\mathrm{s}}^{r}$ than the present approach.

In common with the gradual insertion methods I and III, the constrained cluster algorithm requires (in general) knowledge of a set of weights for its operation. However, because the method focuses on free energy differences, the typical number of overlaps $\bar{N}_{\mathrm{o}}$ is generally far fewer than encountered in methods I and III, and hence the degree of weighting required to reach $N_{\mathrm{o}}=0$ is much less. For example, for $\eta_{\mathrm{s}}^{r}=0.32$, and a grid point separation of $x_{i+1}-x_{i}=0.05$ we find $\bar{N}_{\mathrm{o}} \approx 20$ (see Fig. 8) which is to be compared with the $\approx 200$ overlaps that occur for shell insertion in methods I and III. Thus weight calculation is relatively quick and easy for method V, and indeed we find that if we reduce the small particle volume fraction to $\eta_{\mathrm{s}}^{r} \lesssim 0.2$, then no weights are required at all since the system samples the $N_{\mathrm{o}}=0$ state sufficiently of-

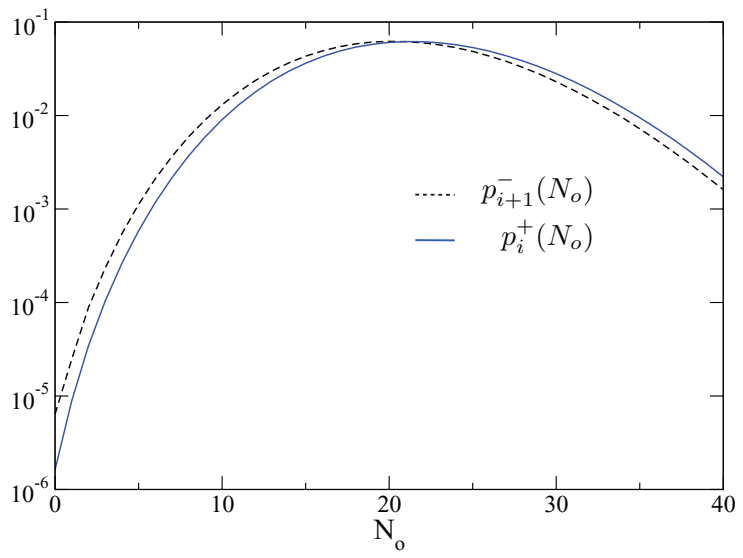

FIG. 8. Estimates of $p_{i}^{+}\left(N_{\mathrm{o}}\right)$ and $p_{i+1}^{-}\left(N_{\mathrm{o}}\right)$ for $i=0$, corresponding to contact of the big particles (i.e., $r_{\mathrm{bb}}=1.0 \sigma_{\mathrm{b}}$ ) as obtained using method V. The grid point separation is $x_{i+1}-x_{i}=0.05$. The ratio of the values of these functions for $N_{\mathrm{o}}=0$ provides an estimate of the difference in the effective potential between the grid points via Eq. (23). ten without the aid of biasing. Even in cases where weighting is required, it is in general not necessary to calculate weights for every grid point; to the extent that the effective potential does not vary strongly between grid points, weights found for one grid point will suffice for all other grid points.

Although it is perhaps reminiscent of methods that obtain the depletion potential by integrating the measured force in a molecular dynamics setting, ${ }^{13-16}$ method $\mathrm{V}$ provides $e x$ act differences in the depletion potential, i.e., no quadrature is required. However, one downside of the need to sum free energy differences to obtain the depletion potential is that cumulative errors arise. The error grows with the number of differences summed and can potentially lead to an estimate for $W\left(r_{\mathrm{bb}}\right)$ that whilst appearing quite smooth, nevertheless deviates significantly from the exact form. Since we commence summing the free energy differences at large $r$, where the potential can be assumed to be essentially zero, this implies that the largest errors occur near contact. To be more precise, for $j=1 \cdots N$ free energy differences, the variance in the sum is simply the sum of the variances of the individual (uncorrelated) estimates, i.e., $\sigma_{N}^{2}=\sum_{j=1}^{N} \sigma_{j}^{2}$. If each individual measurements receives an equal computational expenditure then to a good approximation the cumulative error after summing $N$ differences is simply $\sigma_{N}=\sqrt{N} \sigma$. This growth in the uncertainty in the estimate of $W(r)$ as $r$ decreases, contrasts with the gradual insertion methods where every point in the estimate of $W\left(r_{\mathrm{bb}}\right)$ is independent.

Finally in this subsection we remark that in common with methods I-III, method V is parallelisable with respect to calculations along the grid: one can simply set up independent copies of the simulated system each of which calculates $W\left(x_{i+1}\right)-W\left(x_{i}\right)$ for a different grid point $i$.

\section{DISCUSSION}

In Secs. IV and V we have described five distinct methods for determining depletion potentials in highly size asymmetrical hard sphere mixtures. We now turn to a discussion of their relative merits.

Let us begin by comparing the gradual insertion methods I-III amongst themselves. In terms of their relative efficiency, we find that once prepared so that sampling can commence, each of the methods I-III take a similar amount of CPU time to achieve a given statistical accuracy for $W\left(r_{\mathrm{bb}}\right)$. This is shown in Fig. 9(a) which displays the form of the depletion potential at $\eta_{s}=0.32$ for $q=0.1$ as obtained from methods I-III. The same amount of CPU time ( $35 \mathrm{~h}$ per point on a $2 \mathrm{GHz}$ processor) was invested in each method, and the curves are comparable with respect to smoothness. This finding is perhaps not surprising since in one way or another they all seek to bias small particles out of the way so that one can calculate the insertion probability of a big hard shell.

However, when one takes into account computational startup costs, significant differences arise in the overall efficiencies of methods I-III. Method I is the most cumbersome of the three in this respect since it requires both the choice of a suitable set of staged intermediates and knowledge of a set of weight factors to facilitate transitions between them. Methods II and III remove one or other of these obstacles. 
Specifically, method II entails the choice of staged intermediates, but needs no weight factors, while method III dispenses with staged intermediates, but requires weights. The task of obtaining weights can be relatively time consuming (though largely intervention free if one uses automated techniques such as the TMMC method of the Appendix). The choice of a suitable set of stages is somewhat less time consuming in comparison and can also be easily automated, but is nevertheless cumbersome. This, combined with the need in method II to perform multiple simulation at each value of $r_{\mathrm{bb}}$, renders it slightly inferior to method III, in our view. That said, and at the end of the day, whether one chooses to use method II or III is probably as much a matter of personal taste than of efficiency.

In terms of the domain of applicability of the gradual insertion approach, methods I-III, we note that all three methods are effective in facilitating estimates of depletion potentials at rather high volume fractions of small particles. In this paper we have presented results for systems having $\eta_{\mathrm{s}}^{r} \lesssim 0.32$ and the size ratio $q=0.1$. Elsewhere ${ }^{8}$ we have shown that gradual shell insertion operates effectively up to about $\eta_{\mathrm{s}}^{r}=0.35$. This limit arises from a rapid increase in the relaxation time for the small particles which are tightly packed at this volume fraction. For size ratios smaller than $q=0.1$, the problem of determining the depletion potential is certainly computationally harder than for $q=0.1$ because the typical number of overlaps $N_{\mathrm{o}}$ between the shell and the small particles is greater. Nonetheless we still expect, in principle, to be able to reach small particle volume fractions of $\eta_{\mathrm{s}}^{r} \approx 0.35$.

Methods I-III all construct the depletion potential from measurements of $W\left(r_{\mathrm{bb}}\right)$ across a set of values of $r_{\mathrm{bb}}{ }^{43}$ Since each such measurement is independent, this has the attractive feature that there are no correlations among the data points that form the estimate of $W\left(r_{\mathrm{bb}}\right)$. However, a potential disadvantage of the approach arises from the fact that $W\left(r_{\mathrm{bb}}\right)$ is obtained as the difference of two measurements, i.e., $W\left(r_{\mathrm{bb}}\right)=\ln p_{i}(\infty)-\ln p_{i}\left(r_{\mathrm{bb}}\right)$. In general, both $\ln p_{i}(\infty)$ and $\ln p_{i}\left(r_{\mathrm{bb}}\right)$ are large compared to their difference, and thus, in effect, the gradual insertion approach calculates a small number by subtracting measurements of two large ones. Accordingly for a given fractional uncertainty in $\ln p_{i}\left(r_{\mathrm{bb}}\right)$, the corresponding fractional uncertainty in $W\left(r_{\mathrm{bb}}\right)$ is larger by a factor of $\sqrt{2} \ln p_{i}\left(r_{\mathrm{bb}}\right) / W\left(r_{\mathrm{bb}}\right)$, requiring a greater computational effort to obtain a satisfactorily smooth estimate of $W\left(r_{\mathrm{bb}}\right)$. To give this issue some scale, for the case $q=0.1, \eta_{\mathrm{s}}^{r}=0.2$ we find that $-\ln p_{i}\left(r_{\mathrm{bb}}\right) \approx 200$ for shell insertion (while incidently, $-\ln p_{i}\left(r_{\mathrm{bb}}\right) \approx 600$ for sphere insertion). These are to be compared with the maximum variation in $W\left(r_{\mathrm{bb}}\right)$ of $<4$. Increasing $\eta_{\mathrm{s}}^{r}$ to 0.32 gives for shell and sphere insertion, values of $-\ln p_{i}\left(r_{\mathrm{bb}}\right) \approx 450$ and $-\ln p_{i}\left(r_{\mathrm{bb}}\right) \approx 1700$, respectively, to be compared with a maximum variation in $W\left(r_{\mathrm{bb}}\right)$ of $\approx 8$.

This consideration led us to consider the utility of methods that measure the depletion potentials by focusing on the differences in the potential as the big particle separation is varied. Specifically we have assessed two cluster algorithms. The GCA (method IV) is efficient in the regime of low $\eta_{\mathrm{s}}^{r} \lesssim 0.2$ and represents the method of choice in this range, delivering accurate and efficient estimates of depletion potentials without startup costs or the need for biased sampling. However, to go beyond the rather limited range of small particle volume fractions for which the GCA operates, the constrained cluster algorithm (method V) seems a useful tool. It can attain values of $\eta_{\mathrm{s}}^{r}$ as large as those accessible to the gradual insertion methods. However, a caveat is that the apparent smoothness of the estimates of $W(r)$ arising from method $\mathrm{V}$ may belie the true absolute error in $W(r)$, which accumulates from large to small values of $r$. Our tests in the high density regime (cf. Fig. 9(b)), show that for a given expenditure of computational effort the maximum statistical error in the potential obtained from method $\mathrm{V}$ is comparable, but not significantly superior to the gradual insertions methods. However, there is scope for further improving the efficiency of method $\mathrm{V}$ by using a pair of spherical caps rather than a spherical shell for the subvolume in which preferential updating of small particles is performed. Such subvolumes would include a higher proportion of the small particles that are effected by the virtual move and thus increase the rate of fluctuation in $N_{\mathrm{o}}$.
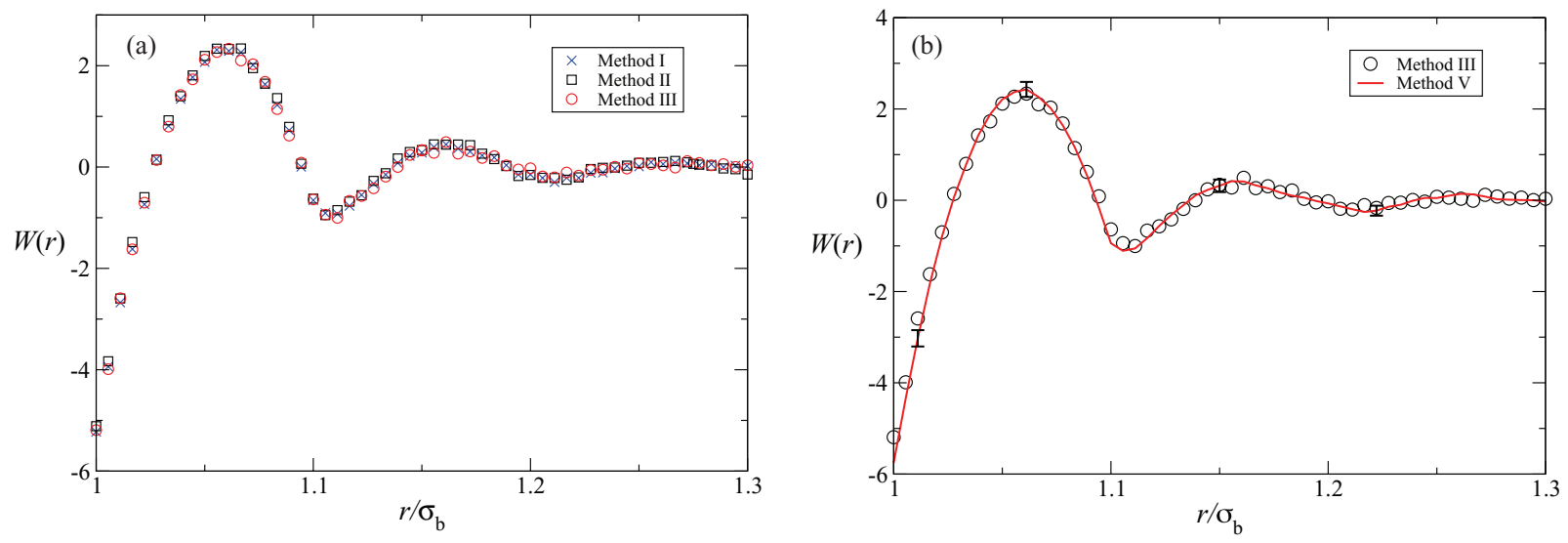

FIG. 9. Estimates of the form of $W\left(r_{\mathrm{bb}}\right)$ for $q=0.1, \eta_{s}^{r}=0.32$. (a) Comparison of the results of methods I-III. (b) Comparison of the results of methods III and V. All data correspond to a computational investment of $35 \mathrm{CPU}$ hours per point on a $2 \mathrm{GHz}$ processor. For methods I-III statistical errors are comparable with symbol sizes. For method V, the statistical error accumulates in going from large to small $r$ as indicated by the representative error bars. 


\section{SUMMARY AND OUTLOOK}

In summary, we have investigated a number of simulation techniques that facilitate accurate measurements of depletion potentials in highly size asymmetrical mixtures of hard spheres. Two categories of approach were considered: (i) gradual insertion and (ii) cluster methods. In the first category, three flavors of methods were described all of which obtain the depletion potential via measurements of the insertion probability of a big sphere or shell in the neighbourhood of another big sphere. Once prepared so that sampling could begin, all three insertion methods showed comparable efficiency. However, differences were found in the startup costs associated with factors such as whether the respective methods require precalculation of staged intermediates and/or weight factors. The gradual insertion methods allow one to obtain depletion potentials for small particle volume fractions of up to about $\eta_{\mathrm{s}}^{r}=0.35$. However to reach this limit it is essential to employ geometrical shortcuts, namely, shell insertion and preferential sampling of small particles in the neighbourhood of a big one. We remark that gradual insertion techniques have recently been extended to systems containing many big particles in a full grand canonical ensemble simulation scheme for highly size asymmetrical fluid mixtures. ${ }^{32}$

In the second category, two cluster algorithms were considered: the geometrical cluster algorithm and a bespoke constrained cluster method. The GCA is very efficient provided $\eta_{\mathrm{s}}^{r} \leq 0.2$. The constrained cluster algorithm considerably extends the range of $\eta_{s}^{r}$ for which depletion potentials can be calculated to at least $\eta_{\mathrm{s}}^{r} \approx 0.32$, albeit at the price of the need to calculate a weight function for use in biased sampling. This makes it competitive with gradual insertion algorithms, though the hope that it would be considerably superior in terms of overall efficiency was not borne out.

Finally we note that with the exception of the shell trick, all the methods considered here can be straightforwardly extended to deal with size asymmetrical mixtures of particles interacting via more general potentials. For the gradual insertion methods, the relevant observable is not the number of overlaps but the energy of overlap, as has already been described in the context of a grand canonical staged insertion study of a highly size asymmetrical Lennard-Jones fluid similar to method I. ${ }^{32}$ For the cluster methods, a version of the GCA suitable for arbitrary potentials is well known. ${ }^{38}$ The constrained cluster method could similarly be easily extended to arbitrary interactions by considering the energy associated with the trial move and measuring the ratio of acceptance probabilities for the forward and reverse move. Of course dealing with longer range interactions comes at a computational price, but the main computational bottleneck, namely that which arises from the high degree of size-asymmetry, should nevertheless be addressed by our methods.

\section{ACKNOWLEDGMENTS}

This work was supported by EPSRC Grant Nos. EP/I036192 and GR/F047800 and the Visiting Postgraduate Scholar Programme of the University of Bath. Some of the simulations were performed on a computer funded by the
HEFCE infrastructure fund. V.S.G. gratefully acknowledges the support of CSIC and a JAE program Ph.D. fellowship from the Dirección General de Investigación Científica y Técnica under Grant No. FIS2010-15502 and from the Dirección General de Universidades e Investigación de la Comunidad de Madrid under Grant No. S2009/ESP/1691 and Program MODELICO-CM. We thank Rob Jack and Bob Evans for useful conversations.

\section{APPENDIX: TRANSITION MATRIX MONTE CARLO}

The choice of method for determining the weight function that allows the system to sample states of low probability states is to some extent a matter of personal taste. A number of approaches exist such as the Wang-Landau method ${ }^{44}$ or successive umbrella sampling. ${ }^{45}$ In this work, we have found the transition matrix method ${ }^{46}$ to be a particularly efficient means of determining a suitable weight function. The transition matrix method has the attractive feature that the weights can be updated "on the fly" throughout the simulation, allowing the simulation to explore an ever wider range of states as the weight function evolves, until it eventually encompasses the state in which a particle or shell is fully inserted. Once this has been achieved, one can cease updating the weight function and perform a production run with a constant weight function.

The general idea of the transition matrix method for determining weight functions is to record the acceptance probabilities of all attempted transitions and extract the ratio of the states' probabilities from it. As all attempted transitions contribute to the weight function, including those that were rejected, the weight function can be built up rather quickly. An outline of the implementation is summarized below, while further details have appeared elsewhere. ${ }^{46-48}$

To implement the transition matrix method, one first defines an order parameter $M$, for which a weight function is desired. For example, in methods III and IV, $M$ would represent the number of overlaps $N_{\mathrm{o}}$ whereas in method I it is the index $m$ of the stage. Then, for every attempted update, the acceptance probability $p_{a}$ (which is calculated anyway for use in the Metropolis criterion) is stored in a collection matrix $C$ :

$$
C\left(M \rightarrow M^{\prime}\right) \Rightarrow C\left(M \rightarrow M^{\prime}\right)+p_{a} .
$$

At the same time, the probability for rejecting the move and thereby keeping the current value of the order parameter is also stored:

$$
C(M \rightarrow M) \Rightarrow C(M \rightarrow M)+\left(1-p_{a}\right) .
$$

It is important to note that these probabilities $p_{a}$ are the "bare" acceptance probabilities and do not include any weights. Thus for insertions and deletions of small hard spheres, as in the present work, they are simple zero or unity.

The transition probabilities are then calculated by normalizing the collection matrix:

$$
T\left(M \rightarrow M^{\prime}\right)=\frac{C\left(M \rightarrow M^{\prime}\right)}{\sum_{k} C\left(M \rightarrow M_{k}\right)},
$$

with the sum on the right-hand side including all possible states to which the system can jump from a given state. In 
the most general case, this would create an $N \times N$ "transition matrix," $N$ being the number of values of the order parameter $M$ to be sampled. The desired probability distribution $p(M)$ of the order parameter follows as the eigenvector corresponding to the unit eigenvalue. ${ }^{46}$ However, in many cases, such as the methods described in the present paper, transition takes only unit steps in the order parameter $M$, implying that the transition matrix is tridiagonal. It follows that $p(M)$ can be constructed simple from the ratio of the probabilities of two adjacent values of $M$ :

$$
\frac{p\left(M_{i+1}\right)}{p\left(M_{i}\right)}=\frac{T\left(M_{i} \rightarrow M_{i+1}\right)}{T\left(M_{i+1} \rightarrow M_{i}\right)},
$$

yielding the weight difference

$$
\begin{aligned}
w\left(M_{i+1}\right)-w\left(M_{i}\right) & =-\ln \left(\frac{p\left(M_{i+1}\right)}{p\left(M_{i}\right)}\right) \\
& =-\ln \left(\frac{T\left(M_{i} \rightarrow M_{i+1}\right)}{T\left(M_{i+1} \rightarrow M_{i}\right)}\right) .
\end{aligned}
$$

Thus, by accumulating the transition matrix in the course of a simulation, one obtains an estimate for $P(M)$ which can be used to update the weight function $w(M)$, thereby allowing the simulation to explore a wider range of $M$. Repeated updates of $w(M)$ extend systematically the range of $M$ over which statistics for the weight function are accumulated, until ultimately the simulation samples' states in which the big particle is fully inserted. However since updating the weight function during a simulation violates detailed balance, we chose to do this at rather infrequent intervals of 20000 sweeps. Once the transition matrix includes value of $M$ corresponding to the fully inserted state, the associated estimate of $p(M)$ provides a measure of the insertion probability, as explained in Secs. IV A and IV B.

${ }^{1}$ C. N. Likos, Phys. Rep. 348, 267 (2001).

${ }^{2}$ L. Belloni, J. Phys.: Condens. Matter 12, R549 (2000).

${ }^{3}$ H. N. W. Lekkerkerker and R. Tuinier, Colloids and the Depletion Interactions, Lecture Notes in Physics Vol. 833 (Springer, Berlin, 2011).

${ }^{4}$ J. C. Crocker, J. A. Matteo, A. D. Dinsmore, and A. G. Yodh, Phys. Rev. Lett. 82, 4352 (1999).

${ }^{5}$ V. Boţan, F. Pesth, T. Schilling, and M. Oettel, Phys. Rev. E 79, 061402 (2009).

${ }^{6}$ B. Götzelmann, R. Evans, and S. Dietrich, Phys. Rev. E 57, 6785 (1998).

${ }^{7}$ R. Roth, R. Evans, and S. Dietrich, Phys. Rev. E 62, 5360 (2000).
${ }^{8}$ D. Ashton, N. Wilding, R. Roth, and R. Evans, Phys. Rev. E 84, 061136 (2011).

${ }^{9}$ M. Oettel, H. Hansen-Goos, P. Bryk, and R. Roth, Europhys. Lett. 85, 36003 (2009).

${ }^{10}$ A. Santos, Phys. Rev. E 86, 040102 (2012).

${ }^{11}$ M. L. de Haro, C. Tejero, and A. Santos, J. Chem. Phys. 138, 161104 (2013).

${ }^{12}$ M. Dijkstra, R. van Roij, and R. Evans, Phys. Rev. E 59, 5744 (1999).

${ }^{13}$ T. Biben, P. Bladon, and D. Frenkel, J. Phys: Condens. Matter 8, 10799 (1996).

${ }^{14}$ R. Dickman, P. Attard, and V. Simonian, J. Chem. Phys. 107, 205 (1997).

${ }^{15}$ B. Götzelmann, R. Roth, S. Dietrich, M. Dijkstra, and R. Evans, Europhys. Lett. 47, 398 (1999).

${ }^{16}$ A. R. Herring and J. R. Henderson, Phys. Rev. E 75, 011402 (2007).

${ }^{17}$ P. Attard, J. Chem. Phys. 91, 3083 (1989).

${ }^{18}$ J. Malherbe and S. Amokrane, Mol. Phys. 99, 355 (2001)

${ }^{19}$ J. Liu and E. Luijten, Phys. Rev. E 71, 066701 (2005)

${ }^{20}$ S. A. Barr and E. Luijten, Langmuir 22, 7152 (2006).

${ }^{21}$ J. Kolafa, S. Labik, and A. Malijevsky, Phys. Chem. Chem. Phys. 6, 2335 (2004).

${ }^{22}$ D. Frenkel and B. Smit, Understanding Molecular Simulation (Academic, San Diego, 2002).

${ }^{23}$ B. M. Mladek and D. Frenkel, Soft Matter 7, 1450 (2011).

${ }^{24}$ B. Widom, J. Chem. Phys. 39, 2808 (1963).

${ }^{25}$ C. Dress and W. Krauth, J. Phys. A 28, L597 (1995).

${ }^{26}$ I. Nezbeda and J. Kolafa, Mol. Simul. 5, 391 (1991).

${ }^{27}$ P. Attard, J. Chem. Phys. 98, 2225 (1993).

${ }^{28}$ N. B. Wilding and M. Muller, J. Chem. Phys. 101, 4324 (1994).

${ }^{29}$ D. Kofke and P. Cummungs, Mol. Phys. 92, 973 (1997).

${ }^{30}$ A. D. Bruce and N. B. Wilding, Adv. Chem. Phys. 127, 1 (2003).

${ }^{31}$ A. P. Lyubartsev, A. A. Martsinovski, S. V. Shevkunov, and P. N. Vorontsov-Velyaminov, J. Chem. Phys. 96, 1776 (1992).

${ }^{32}$ D. J. Ashton and N. B. Wilding, Mol. Phys. 109, 999 (2011).

${ }^{33}$ R. W. Zwanzig, J. Chem. Phys. 22, 1420 (1954).

${ }^{34}$ J. R. Errington and D. A. Kofke, J. Chem. Phys. 127, 174709 (2007).

${ }^{35}$ C. Bennett, J. Comput. Phys. 22, 245 (1976).

${ }^{36}$ N. Lu, J. K. Singh, and D. A. Kofke, J. Chem. Phys. 118, 2977 (2003).

${ }^{37}$ K. Ding and J. Valleau, J. Chem. Phys. 98, 3306 (1993).

${ }^{38}$ J. Liu and E. Luijten, Phys. Rev. Lett. 92, 035504 (2004).

${ }^{39}$ J. Liu, N. Wilding, and E. Luijten, Phys. Rev. Lett. 97, 115705 (2006).

${ }^{40}$ D. Ashton, N. Wilding, and P. Sollich, J. Chem. Phys. 132, 074111 (2010).

${ }^{41}$ D. Ashton, J. Liu, E. Luijten, and N. Wilding, J. Chem. Phys. 133, 194102 (2010).

${ }^{42}$ J. G. Malherbe and W. Krauth, Mol. Phys. 105, 2393 (2007).

${ }^{43}$ Note also that methods I-III permit direct estimates of the contact value of the depletion potential. This contrasts with methods that obtain the depletion potential by integrating the force, which rely on extrapolation to estimate the contact value.

${ }^{44}$ F. Wang and D. Landau, Phys. Rev. E 64, 056101 (2001).

${ }^{45}$ P. Virnau and M. Müller, J. Chem. Phys. 120, 10925 (2004).

${ }^{46} \mathrm{G}$. Smith and A. Bruce, J. Phys. A 28, 6623 (1995).

${ }^{47}$ J. Errington, J. Chem. Phys. 120, 3130 (2004).

${ }^{48}$ G. McNeil-Watson and N. Wilding, J. Chem. Phys. 124, 064504 (2006). 